\title{
JOB-EMBEDDED, COLLABORATIVE AND REFLECTIVE PROFESSIONAL DEVELOPMENT FOR UNIVERSITY ADMINISTRATORS: THE ACTION LEARNING PEDAGOGY
}

\author{
R. Middlehurst \\ Kingston University and Higher Education Academy \\ London, United Kingdom \\ e-mail: R.Middlehurst@kingston.ac.uk
}

\section{Cross*}

Faculty of Education and Ali Mazrui Centre for Higher Education Studies

e-mail: mcross@uj.ac.za

\section{Jeannin*}

Faculty of Education and Ali Mazrui Centre for Higher Education Studies

e-mail: loisej@uj.ac.za

*University of Johannesburg

Johannesburg, South Africa

\section{ABSTRACT}

Job-embedded, collaborative, and reflective professional development programmes have generally been praised internationally for promoting knowledge sharing and meeting the learning needs of extremely busy practitioners, such as university academic and administrative staff. However, in the Southern African context where, for a variety of reasons, professional development draws extensively on traditional pedagogies, their usefulness has not been fully tested. Analysing the experiences of 11 participants of the Programme for University Leadership in the Southern African Region (PULSAR) and subsequent developments within their own institutions, this article shows how Action Learning can be used as a tool for change for university senior administrators. Through this job-embedded, collaborative, and reflective pedagogy, Action Learning provides enabling conditions for university administrators to unlearn, learn and relearn engagement strategies (e.g., questioning and listening skills, participative team-work) to approach workplace problems differently, and in the process, build more effective working relationships.

Keywords: action learning, professional development, university administrators, pedagogy for adult learners, collaboration, participative leadership.

\section{INTRODUCTION}

This article explores the pedagogy of Action Learning (AL), a method for problem-solving and 
learning from collaboration, as it was used and experienced in the Programme for University Leadership in the Southern African Region (PULSAR) in 2012. This programme was designed by the Southern African Regional University Association (SARUA) in partnership with the United Kingdom's (UK's) Leadership Foundation for Higher Education, and offered to university registrars and those with senior administrative functions in higher education institutions in the 15 countries of the Southern African Development Community (SADC region). Eighteen participants from 17 institutions in eight countries participated in the programme. The article reviews how the AL programme was implemented and how it was subsequently unfolded at various institutions. It commences with an outline of the key features of the AL pedagogy, followed by a description of the PULSAR Programme and a detailed analysis of the learning experiences of the participants.

Within the SADC region, each country and institution has its particular history and developmental trajectory. In addition, participants in the PULSAR Programme had their own professional objectives at their distinctive institutions. Within this complexity, the AL pedagogy was intended to assist administrators in meeting those objectives by applying the methodology to their institutional projects. For most of the participants, the experience represented their first encounter with the AL method.

This article critically reviews the experiences of administrators in using the $\mathrm{AL}$ methodology during the workshops and within their varied institutional and national contexts, looking at both the achievements of the programme, which are overwhelmingly positive, and the areas that require development and adaptation in future endeavours. It argues that the jobembedded, collaborative, and reflective characteristics of the AL pedagogy enabled participants to develop questioning and listening skills that could prove useful in their daily work. The PULSAR experience suggests that AL takes into account the specific individual and group profiles of participants, and the contextual complexity in which they operate. Hence, the article argues that when combined with adequately conceptualised delivery, the AL approach could be an important pedagogical device in leadership development programmes for senior university administrators in the context of Southern Africa.

\section{ACTION LEARNING: A JOB-EMBEDDED, COLLABORATIVE, AND REFLECTIVE PEDAGOGICAL APPROACH}

Three important epistemological and theoretical insights from the literature on AL appear central to understanding its particular process: (i) its job-embeddedness suitable for situated learning; (ii) its emphasis on collaborative learning; and (iii) its reflective and reflexive 
dimensions. The integration of these three dimensions coupled with sensitivity to the contextual complexities of the region underpinned the pedagogical approach adopted in the PULSAR programme.

\section{A job-embedded approach suited for situated learning}

The first defining aspect of AL pedagogy concerns the nature of knowledge and learning in context. For this particular aspect, the article draws on Brumer's (as cited in Brown and Duguid 2000) distinction between the notions of learning about and learning to be. The former is concerned with learning facts, concepts and procedures, often through the transmission mode which, although it comprises most of the learning in education institutions, is not sufficient for developing effective ways of seeing and engaging with the world as knowledge practitioners. The latter, learning to be conceptualises learning as becoming a practitioner of knowledge in a professional domain (e.g., university registrarship) to enable participants to act purposefully in situations they are going to encounter in the future. It requires mastery of the practices of knowledge in a discipline, including the principles, competencies, procedures and values in a professional field. This is important in a world such as Southern Africa and Africa in general, marked by what Barnett (2000) refers to as 'supercomplexity', or a 'world without stable meanings ... in which the handling of uncertainty, ambiguity and contestability comes to the fore’ (Barnett and Hallam 1999, 145).

University administrators in such a complex environment find themselves overwhelmed by an array of activities and daily routines that leave limited space for learning beyond their jobs. Contrary to traditional professional development programmes, PULSAR activities seem to have given primacy to learning to be, without neglecting the required dimensions of learning about. Participants were not just learning theory about their work separated from themselves, they were also creating new understandings to generate changes, while reflecting on their actions and concurrently learning about themselves (Spraker 2007, 4).

\section{Collaboration as a trigger for social change}

Drawing on the situated cognition theory (Brown, Collins and Duguid 1989) and communities of practice (Lave and Wenger 1991; Wenger 2000; 2011), collaborative learning can provide ideas, support, and feedback to keep up with university changes and critically reflect on one's practice. The AL pedagogy was based on collaborative enquiry to draw on peers' questions and deepen one's understanding of job-embedded issues. Hence, the second theoretical background underpinning AL pedagogy emphasises cooperative social problem-solving strategies and 
brings individual transformation within the context of the group. Within this framework, diagnosis of problems goes beyond constructing a problem by building individual and collective commitment to action and change. As Weisbord $(2004,94)$ put it, 'we are likely to modify our own behavior when we participate in problem analysis and solution and are likely to carry out decisions we have helped to make'. Therefore, the AL pedagogy provides selfdirected learning opportunities building upon participants' intrinsic motivation by allowing them to customise group discussions to their own needs. Participants ask questions on practical issues on site and enable their peers to reflect on their roles and assumptions in the specific organisation.

\section{Questioning, reflectivity and reflexivity}

The third aspect of AL concerns targeted questioning, reflectivity and reflexivity as methodological concepts which, though not made explicit in the methodology, are important analytical tools for understanding this particular process. Targeted questioning as a method could certainly claim dimensions of the well-known Socratic midwifery or generative pedagogy in which learning is facilitated through questioning that leads to reflection and better understanding of the consequences of one's actions. Reflexivity requires relentless selfquestioning of the way one does things and constructs meaning while acting and interacting. It entails acute self-awareness of one's assumptions and practices as translated in the double loop of reflection on action and reflection in action. On the contrary, reflectivity pertains to the retrospective in-depth analysis applied to past experiences (Ryan 2005). Hence, one does not learn automatically through experiences or actions. It is only through reflective skills that one becomes aware of assumptions and beliefs, and how these may influence perceptions and behaviours. Being reflective is thus a condition for becoming reflexive, being able 'to suspend our beliefs and perceptions as the reality' and starting 'to "see” from other perspectives' (Spraker 2007, 9-10).

\section{METHODOLOGY}

Besides data obtained through observations and direct interactions with the administrators, this study is based on group and individual interviews with the participants. Individual interviews and a focus group were held with 11 participants to learn about their experiences and reflections on the AL process. Three research questions have been designed to investigate participants' encounters with AL: 
- How did participants experience AL throughout and after the PULSAR Programme?

- To what extent did participants use AL at their respective institutions? And in the management of their distinctive project?

- What challenges or benefits did they experience in using AL?

Content thematic analysis has been applied to the group and individual interviews' transcripts to elicit the prominent themes and characterise participants' experiences with AL.

\section{CONTEXT}

The search for suitable pedagogies for leadership development for senior university administrators remains a major challenge in Southern Africa. The first university association in the region, the South African Universities Vice-Chancellors Association (SAUVCA) was defined to provide policy support to university vice-chancellors. Professional development was inaugurated by the Tertiary Education Linkages Programme in South Africa (TELP 19952004), which, besides supporting government policy development, aimed at enabling historically disadvantaged institutions to deal with management, leadership and curriculum issues through partnerships and linkages with American universities. The Centre for Higher Education Transformation (CHET) introduced training workshops on similar issues in 19992003, whilst the Council for Higher Education (CHE) also arranged capacity development workshops and consultative meetings with senior administrators. At a regional level, this role has been played by the Association of African Universities (AAU) and the Council for the Development of Social Science Research in Africa (CODESRIA), and more recently by SARUA. While conversations with peers and input by experts in seminars and conferences appear to have been beneficial, a neglected area has been the search for suitable pedagogues and pedagogies that fit the profiles of university administrators. These senior administrators are adult learners with rich experiences and considerable knowledge about the field in which they operate. The PULSAR Programme, building on the AL pedagogy, appears to be one of the most significant experiments in this respect.

However, the use of AL in Southern Africa faces several challenges. It must confront the long-enduring colonial and apartheid legacies entrenched in society and institutions manifested in the culture of authoritarianism and autocratic decision-making. Worth mentioning also are forms of individualism promoted by recent market and neo-liberal discourses that emphasise competition and performativity, leaving little room for collaboration. In such environments, AL, with its emphasis on questioning and individual agency, tends to be received with a degree 
of scepticism, as articulated by one of the participants:

It is more of a challenge to use this method because of the sense of individualism in the university and because of needing to 'appear good in front of the boss' ... the culture is more competitive than collaborative ... and is not always open and honest; individuals often have a second agenda ... One therefore needs caution in day-to-day dealings with others in the university.

This may explain why, whilst AL has been embraced for several years in business managerial practices in Southern Africa and beyond, it has hardly penetrated the universities' administrative circles in the way it did in the PULSAR Programme.

\section{ACTION LEARNING: ORIGINS AND METHODOLOGY}

The term and the pedagogy of 'Action Learning' were first used by Reginald Revans, a pioneer in management education (Trehan and Pedler 2011). Revans attributes its development to a formative period of his life when he worked with eight Nobel Prize winners at Cambridge University in the UK. Each week during 1928, a group of physicists met to discuss their experiments, with a focus on trying to understand each other's difficulties and on learning from each other so that they could work towards a solution for the particular problems they were facing.

This experience reflects the essence of AL: A group of peers, each seeking to bring about some changes in the world, who meet regularly to discuss the difficulties each of them is experiencing. Participants can then test the ideas arising from their discussions. The key pillars of AL were summarised by Revans (1980) through the formula $\mathrm{L}=\mathrm{P}+\mathrm{Q}$ (where $\mathrm{L}$ is learning, $\mathrm{P}$ is programming and Q is questioning to create insight into what people see, hear or feel). The formula was to be later expanded by Michael Marquardt as $L=P+Q+R$, where R refers to reflection. In Marquardt's view, reflection indicates that good questioning should evoke thoughtful reflections around problems (Marquardt et al. 2009). It enables unlearning of bad behaviours and practices, relearning and learning through self-reflection, and systematic engagement with one's established constructs, experiences or practices.

Unlike active learning, which is a pedagogical approach based on participants' actions during the training session (Desimone 2011), AL draws on peer discussions with actions taken outside the sphere of the learning group. AL also differs from problem-based learning (Bridges and Hallinger 1996), as AL group members do not design solutions to specific problems nor answer peers' questions. Instead, they encourage each peer to deconstruct challenges, through questioning and active listening, to help each participant explore the issues from different angles (Marquardt et al. 2009). 
AL is usually practised through groups, consisting of four to eight people, each working on a separate project or challenge, who meet regularly to discuss the problems they encounter with the intention of learning from each other, just as the Cambridge scientists had done (Marquardt et al. 2009). As such, they are similar to the communities of practice conceptualised by Wenger (2000; 2011), although they rely on a specific questioning approach to facilitate peer investigation and growth. The meetings may be facilitated by an external person who can help the group establish ground-rules and structures for working together (such as confidentiality, logistics of meetings, and participation discipline of the AL process).

At each group meeting the time is usually split equally so that everybody focuses on the issues brought by each person. Participants usually present a particular challenge they are facing in relation to the institutional projects for which they are responsible. Meetings have a clear structure to ensure that each individual has time to outline the individual project and key issues guided by others' questions. These questions, which can be closed (e.g., who? what?), objective (e.g., how much or how many?), relative (e.g., where? when?) or open, are a key part of the methodology as they enable exploration of many facets of a problem. The purpose is for group members to assist each individual through asking questions that may be quite different from those one might ask oneself. The group members do not comment or advise on a solution, nor do they give examples of how they would tackle the problem. They rather provide insights through targeted questioning and probing. The tone of each meeting should be open and supportive. A parallel part of the method involves active listening, so that issues can be explored thoughtfully and constructively.

While the AL meeting is a core part of the process, its other essential part is the testing of ideas in action after group meetings. Subsequently, the group helps each individual to reflect on the outcomes of their recent actions and learn from experiences. Through reflection, participants can develop ideas to overcome obstacles. In essence, AL is about learning from action and action informed by learning. Taking responsibility for solving one's own problems and being pro-active in finding solutions is key to success, greatly assisted by the support offered by the group of peers during or between meetings.

\section{THE ACTION LEARNING PEDAGOGY WITHIN THE PULSAR PROGRAMME}

There were several reasons for including AL as part of the design of the PULSAR Programme. First, it made sharing experiences feasible and practically useful for university administrators at different levels of seniority. Second, it was important that the PULSAR Programme focused on practical outcomes as the programme aimed to prompt positive changes while taking into 
consideration the individual profiles and the diverse institutional contexts of the participants. The institutional project that each individual chose was key to ensuring that the programme would yield practical outcomes, and the AL methodology represented a useful vehicle for achieving those outcomes. Third, an aim of the PULSAR Programme was to build networks across the SADC region and the AL process contributed to this networking purpose within the scattered community of administrators.

The use of institutional projects ensured that the commitment to change was not abstract and removed from practice, but rooted in participants' own individual and institutional needs and contexts. The logic of AL activities also acknowledges and legitimises the participants' experiences as a learning basis while moving beyond initial representations through constant self-reflection. As stated by a participant: 'You ... put on the table your thoughts and it produces innovation and new learning'.

Participants were given the opportunity to receive inputs from experts, to participate in group or individual consultations with their group facilitator, and to attend institutional visits where possible. While AL constituted the main pedagogy, the programme included a number of guest speakers' lectures from experienced practitioners in senior university management in the SADC region. These inputs enabled participants to engage in critical discussions.

Lastly, the programme paid attention to overnight reflection on daily activities which were shared in the opening morning sessions. These sessions were deemed useful to maximise individual and group reflectivity and prompt self-reflective learning.

\section{THE ACTION LEARNING PROCESS IN PRACTICE}

The AL process was introduced to participants via a Skype presentation, which outlined the principles of AL, ground-rules for the groups' work (including questioning rules to enact paradigm shift and learning) and information to guide the choice of institutional projects. A particular issue to consider was whether AL groups could meet physically or virtually between the main two workshops, separated by about five months.

The design of the first workshop ensured that plenary sessions were interspersed each day with project-work in AL sets of four or five people, supported by a facilitator. The first workshop allowed participants to build relationships with their colleagues and gain a more thorough understanding of each other's contexts. Facilitators were particularly helpful at this early stage in encouraging participants to adopt the AL methodology and practise targeted questioning and active listening. They provided time and space for participants to outline their projects and associated challenges, and created opportunities for group members to ask probing 
questions.

Two of the groups met between the July and November workshops, but all kept in contact with their group members (electronically and by telephone). The facilitators played a lesser role between workshops, as participants had to work on their projects and provide mutual support. During the second workshop, the time spent in AL sets was focused on preparing project reports against a template and in discussing how the AL process had worked for individual participants during the workshops, in relation to the delivery of their projects and in their wider role within their institutions. In the following sections, the experiences of participants in relation to the $\mathrm{AL}$ process are scrutinized.

\section{THE EXPERIENCES OF PARTICIPANTS IN RELATION TO THE ACTION LEARNING PROCESS}

Some of the comments offered by participants give an insight into the issues they came across when they started using the AL process within their groups and institutions. They progressively developed a better understanding of the key components of the AL pedagogy, namely targeted questioning, active listening, testing of ideas, peer support, and reflection on actions entailed as a strategy for effecting changes despite contextual challenges. This AL process appeared suited for a diverse group of adults with considerable experience and knowledge in their specific niche-areas. In the process, a general consensus began to emerge that AL provided adequate shared learning spaces, with suitable dynamics and values to be responsive to their professional challenges and ethics, as articulated by the participants: 'It created an environment where you could express yourself without criticism and correction'.

\section{Learning from the Action Learning groups}

Participants spoke at length about their surprise and enjoyment of AL within their small groups and explained how an atmosphere of openness and willingness to learn from each other had been engendered. It would appear that some of the conditions for success of the AL pedagogy, outlined in the programme, were met in practice, particularly:

- Willingness to be open with each other

- Motivation to take a hard look at themselves and start making changes

- $\quad$ Capacity to reflect on experience and learn from it

- $\quad$ Ability to ask thought-provoking questions and engage in active listening

- $\quad$ Ability to respond constructively and appropriately to the observations of others. 
It appeared that group members engaged in a critical discussion of their pre-established constructs and assumptions about learning. Many participants, who were used to operating with the concept of learning about through knowledge transmission from experts or wise peers, felt the need to shift their attention to becoming practitioners of their own knowledge grounded in their own institution. Thus, by emphasising participants' reflective focus on their practice, facilitated by peers' targeted questions, AL tended to privilege the role of individual agency in the learning process, moving away from the one-way knowledge transmission system. With regards to their encounters of AL in the PULSAR Programme, participants highlighted their experiences around the following constructs:

\section{Action Learning as source of motivation and commitment to change}

The process is self-rewarding and motivating; it is a richer experience by challenging your own paradigms; in the process, you can become more open-minded. It makes you value members of the team as sources of knowledge and ideas. If you ask open questions you get more than you bargained for ... you also think your challenges are unique and it is comforting to see that they are shared by others ... if you have challenges you can get support from the group.

The AL process made me aware that my style required adjustment; the AL group provided a good opportunity to practice. I was taken out of my zone of comfortable operation in terms of personal work and it helped to make me more adaptable to others. It has given me a personal and professional learning experience that was unexpected and deep.

\section{Questioning as a skill}

There was a natural inclination to say how one should do something rather than asking questions. The questions proved a useful discipline that could be applied in wider working life.

When the concept was presented, I thought it was basic and what we do in daily working life but when we tried it in our group - we found the questioning technique was different. We had to ask questions rather than give answers.

In the Action Learning group ... it has brought useful experiences to me ... when you are given a question, you think more critically, think about more options to come up with a better-quality view of the matter ... for example, when introducing a topic ... I thought this was the issue but when questioned, this wasn't the real problem, it was another.

\section{Listening as a skill}

When you want to get information, you should ask questions and gain listening skills. It's a useful tool in the AL group ... therefore we were able to share a lot ... it was useful to share and we learned a lot; we learned from other projects. 
There were lots of benefits ... in terms of developing listening skills and understanding the questions. The questions helped to develop ideas and helped in formulating the project.

\section{Responding constructively to questions}

It is also important to avoid being defensive when being questioned; to avoid defending oneself ... with the group, it became an acceptable way of operating ... each of us tried to live up to the demands of the methodology.

\section{Usefulness of Action Learning in day-to-day work}

Participants reported on the utility of the AL methodology in their day-to-day work in a number of different institutional contexts, from working with students to working with peers and senior managers. They underlined a number of professional benefits arising from using the AL approach. At the individual level, this was surprising and pleasing, and in some cases quite profound in terms of changes in perceptions, behaviours and skills. At the group level, the AL process created a network that provided continued support even after the PULSAR Programme has finished.

\section{To develop a sense of community and ownership in decision making}

As a Section Head, I'll use the technique because it creates a family bond - this works with subordinates over tea, informally.

If you use questions, ownership of the outcome is better than giving prescription.

In day-to-day work ... what comes is more ownership, less resentment and friction with subordinates ... you have consulted through questioning rather than coming up with the answer and solutions. Individuals can come up with solutions; it is exciting and causes one to think logically and critically.

\section{To ask the right questions and collect useful information}

I have used it when chairing meetings of other registrars of the colleges and faculties (as Registrar, I set policy and they implement) ... I've learned not to say 'why?' when something isn't working and instead to say 'what happened'? This way you get more information and less defensiveness ... the Action Learning process was useful, but this is just the beginning ... I will use it with the induction and training of new recruits, in pre- and post-shadowing meetings to bring out 'what happened' and was the outcome anticipated or not?

With the project, I am now able to ask questions well. I use the tool in management - discuss a lot and do a lot of listening; I feel well-equipped now.

I do sessions with faculty managers - in a very devolved system - and instead of 'telling', I'm more aware of using the Action Learning questioning technique ... To ask is more valuable than telling or answering for others. Teaching others to ask questions in this way is useful and also helps to ask very high-order and complex questions ... the real benefit of Action Learning comes from the calibre of the question ... 


\section{To develop a network}

The Action learning process first brought group members close together; now we have networks and linkages between young and seasoned administrators ...

What I liked about the Action Learning groups is that it brought us close together as friends. I never expected this at the beginning ... and professionally, it is now easy to get information from another university if I need it. New plans have come out of the Action Learning process.

The learning that resulted from the programme, in particular the skill of asking questions to elicit information and gain wider perspectives, and the skill of active listening, were found useful in engaging and motivating stakeholders at work. The art of leading through generative questions (as in the Socratic midwifery method) to engage the stakeholders rather than of 'telling' them was also highly valuable in making progress on the individual institutional projects, as presented below.

\section{Usefulness of Action Learning in relation to the institutional projects}

Job-embeddedness is a central feature of the AL process. It addressed one of the key weaknesses of many previous professional development initiatives for senior administrators as it focused on the specific workplace challenges and the potentialities for change. In this regard, the effectiveness of AL is reported below:

\section{Enriching the projects by eliciting ideas and thinking through the process}

The method was helpful in assisting us to formulate projects with the help of colleagues; it opened new dimensions of the project; it helped to make it more distinct and succinct. It worked to have the benefit of experience from colleagues.

It was a positive method for the project work. The Action Learning questioning approach proved quite natural to the nature of the project; it was useful in getting ideas out of the students and the team because I knew them and in projects where new ideas are needed, the questioning helps.

With the project, the questions helped to focus, think through the process; actions then come from the mind instead of ... the Head ...

\section{Negotiating institutional approval and support}

The project was coherent and easy to sell ... I was able to get the support quickly from stakeholders. I used the Action Learning questioning in seeking support from the VC for the project and to sell it to Deans, Heads of Department and also to the ICT and HR departments. This helped to sell the benefits of a new system ... I had anticipated resistance because the project involves a lot of work; but all sections were supportive and forthcoming, listening and questioning skills helped. I asked: 'How do you keep records? What challenges do you currently face? How could there be 
improvements? What might be needed to deliver these?'

It wasn't easy for colleagues to buy into the project; they didn't understand it ... but I kept on talking about it in formal meetings and also with the Deans who are influential ... the view got appreciated and accepted. I asked questions like: 'How would your Faculty feel if you were to leave? What have you done to help someone else progress? Don’t you think it would be helpful if we had a framework for succession?' - it was then important to avoid looking at the issue personally, but as a system.

Emanating from these constructs is the potential of the AL pedagogy to influence the daily decision-making operations at institutional level. The AL approach can help university administrators 'to enhance their capacity to learn and respond to organisational issues more effectively' as they unlearn, learn and relearn collaboratively. As Miller (2003, 21) put it, 'workplace learning interventions need to be accompanied by a planned strategy that encourages managers to work together in a collaborative way on real workplace issues or problems that will have immediate benefits to the managers and the organization'. In this regard, AL appears as a suitable strategy to create a safe environment that encourages administrators to work together in an atmosphere of trust and mutual respect. However, the process of asking questions rather than sharing one's perspective has proved to be challenging under certain circumstances. These challenges are detailed in the next subsection.

\section{Challenges encountered in using Action Learning}

While the overwhelming evidence from the interviews indicates that the AL pedagogy proved beneficial for working together in AL sets, making progress on institutional projects, and enhancing day-to-day working relationships, the experience was not always smooth. Participants reported that asking questions and listening instead of 'telling' took more time, so they had to persevere with the method until they could see the results. They had to learn how to ask the right questions, and indeed to ask questions, rather than commenting or giving answers. The following views highlight how collaborative learning through questioning, and without supplying ready-made answers, was challenging.

\section{Temptation to prescribe}

What I noted is that the experience doesn't come automatically - it needs to be conscious - there is always the temptation to prescribe - but it can be useful if you practice it.

More usually, instead of letting a person think - you tend to prescribe from your own perspective. 


\section{Difficulties to ask thought-provoking questions}

The difficulties are about asking the right questions; how to ask; avoiding appearing judgemental; instead - just seeking information...

I prefer to work independently; the AL group is like having a supervisor ... if I'm not asked the right questions or they are not sharp enough ... it can be frustrating ... from a close colleague, you get more focused questions.

\section{Challenges in the decision-making process}

It gets you to ask a lot of questions and it takes time to reach a decision ... Each has to be convinced before reaching decisions ...

Thus, for many, AL was not always comfortable, either because of individual styles or crosscultural differences. As one participant jokingly mentioned in the corridor: 'First lesson: learn to keep your ideas to yourself'. Although participants came to see AL as an enabling change mechanism at both individual and group levels, they were wary about the somewhat hostile environment in which they had to apply it in their organisation. This is largely because participative management remains an anathema in some institutions. Group dynamics in such environments are deeply constrained by the hierarchical logic. Despite these challenges, administrators made clear that the AL component of the PULSAR Programme enabled them to develop useful skills they could deploy in their institutions, in addition to widening their network across the different Southern African countries.

\section{CONCLUSION}

Two important aspects emerge from the AL experience. First, the AL pedagogy has helped participants conceptualise their institutional activities and enhance their ability to ask powerful questions in a complex professional environment. The AL pedagogy has been useful within the projects and in day-to-day working practices, and it has enabled individual and institutional learning in such a way that surpasses expectations from both the programme designers and participants. Second, it has given participants a network of colleagues across the region that can offer continued support.

Given the diverse profile of university administrators and the multiplicity of their institutional environments, a combination of AL activities and expert advice from experienced practitioners has also proved effective in bringing about learning and institutional changes. Specifically, the strengths of the PULSAR Programme were grounded in its three components including: (i) AL pedagogy which permeated all project-related activities (group work, morning 
and evening reflective reviews); (ii) inputs and discussions with guest speakers; and (iii) individual and group consultations with facilitators. Hence, although AL programmes do not always depend on these three inputs, AL pedagogy could represent an effective approach for leadership development programmes in Southern Africa.

A challenge for the future is how to balance these three main components, namely AL group activities, guest speakers, and consultations, in a way that individual agency is not neglected. It is also about how to structure the guest speaker sessions beyond the traditional transmission model and turn them into productive support of AL activities. Overall, the PULSAR Programme has paid off and a major step has been made towards finding a pedagogical approach best suited to leadership development for senior administrators in the Southern African context.

\section{REFERENCES}

Barnett, Ronald. 2000. Realizing the university. Buckingham: SRHE and Open University Press.

Barnett, Ronald, and Susan Hallam. 1999. Teaching for supercomplexity: A pedagogy for higher education. In Understanding pedagogy and its impact on learning, 137-54. London, UK: Paul Chapman Publishing.

Bridges, Edwin M. and Philip Hallinger. 1996. Problem-based learning in leadership education. New Directions for Teaching and Learning (68): 53-61. doi:10.1002/tl.37219966809.

Brown, John Seely, Allan Collins and Paul Duguid. 1989. Situated cognition and the culture of Learning. Educational Researcher 18(1): 32-42. http://edr.sagepub.com/

Brown, John Seely and Paul Duguid. 2000. The social life of information. Boston, MA: Harvard Business Press.

Desimone, Laura M. 2011. A primer on effective professional development. Phi Delta Kappan 92(6): 68-71. http://pdk.sagepub.com/content/92/6/68.short

Lave, Jean and Etienne Wenger. 1991. Situated learning: Legitimate peripheral participation. Cambridge, England: Cambridge University Press.

Marquardt, Michael J., H. Skipton Leonard, Arthur M. Freedman and Claudia C. Hill. 2009. Action learning for developing leaders and organizations: Principles, strategies, and cases. Washington, DC: American Psychological Association.

Miller, Peter. 2003. Workplace learning by action learning: A practical example. Journal of Workplace Learning 15(1): 14-23. doi:10.1108/13665620310458785.

Revans, Reginald William. 1980. Action learning: New techniques for management. London, UK: Blond and Briggs Ltd. https://www.cabdirect.org/cabdirect/abstract/19821891111

Ryan, Thomas. 2005. When you reflect are you also being reflexive? Ontario Action Researcher 8(1). http://oar.nipissingu.ca/index.htm

Spraker, Barbara. 2007. The power of action learning for global leadership. Women leading the way. http://www.womenleadingtheway.com/womens-leadership-articles.html

Trehan, Kiran and Mike Pedler. 2011. Cultivating foresight and innovation in action learning: Reflecting ourselves; Reflecting with others. Action Learning: Research and Practice 8(1): 1-4. doi:10.1080/14767333.2011.549320.

Weisbord, Marvin R. 2004. Productive workplaces revisited: Dignity, meaning, and community in the 21st Century. San Francisco, CA: Jossey-Bass. 
Wenger, Etienne. 2000. Communities of practice and social learning systems. Organization 7(2): 225246. https://doi.org/10.1177/135050840072002

Wenger, Etienne. 2011. Communities of practice: A brief introduction. https://scholarsbank. uoregon.edu/xmlui/handle/1794/11736 